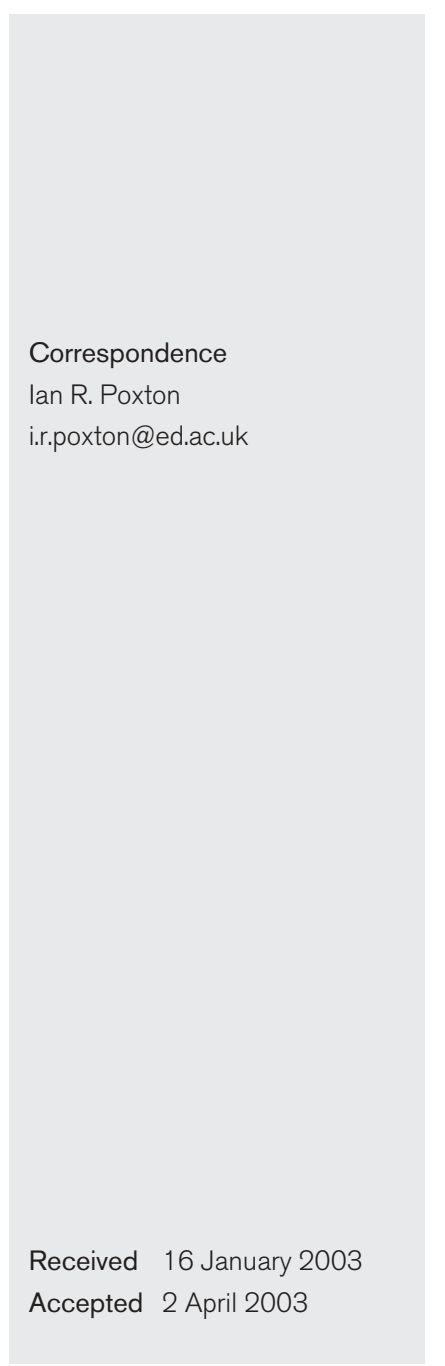

\title{
Clostridium difficile in a geriatric unit: a prospective epidemiological study employing a novel S-layer typing method
}

\author{
Jodie McCoubrey, ${ }^{1}$ John Starr, ${ }^{2}$ Heather Martin ${ }^{2}$ and lan R. Poxton ${ }^{1}$ \\ ${ }^{1}$ Medical Microbiology, Centre for Infectious Diseases, The University of Edinburgh Medical School, \\ Teviot Place, Edinburgh EH8 9AG, UK \\ ${ }^{2}$ Geriatric Medicine Unit, University of Edinburgh, Royal Victoria Hospital, Craigleith Road, Edinburgh \\ EH4 2DN, UK
}

\begin{abstract}
Clostridium difficile is the major identifiable cause of antibiotic-associated diarrhoea in the UK. The aim of this study was to employ traditional culture, toxin detection and a novel typing method to determine the level of $C$. difficile colonization and disease in a population of elderly patients and to investigate the association between strains in the patients and their environment. Three hundred and ninety patients between 62 and 101 years of age admitted to a geriatric unit in the Royal Victoria Hospital (RVH), Edinburgh, were investigated for the presence of C. difficile. C. difficile was cultured from 100 (26\%) patients using pre-reduced cycloserine-cefoxitin egg yolk agar, and toxin(s) was detected in the faeces of 34 of these patients using the Techlab ELISA test kit for the detection of $C$. difficile toxins $\mathrm{A}$ and/or B. Toxin(s) was detected in a further 18 patients from whom no $\mathrm{C}$. difficile was detected in culture. Of the patients in whom C. difficile was detected, $49 \%$ had diarrhoea, with the highest proportion of patients with diarrhoea being both culture- and toxin-positive for C. difficile. Environmental sampling of the patient environment yielded C. difficile from $14 \%$ of samples. The organism was most frequently isolated from floors, sluice-rooms and toilet areas. The variation in the molecular mass of the C. difficile S-layer proteins was exploited as the basis of a novel typing method for $\mathrm{C}$. difficile. Isolates from patients in the RVH were given a four-digit 'S-type' number based on their S-layer protein profile. A total of seven S-types were identified, with one type, toxigenic S-type 5236 , accounting for $73 \%$ of all clinical isolates and $91 \%$ of environmental isolates.
\end{abstract}

\section{INTRODUCTION}

Clostridium difficile is a well-established nosocomial pathogen, responsible for a significant number of cases of antibiotic-associated diarrhoea. C. difficile-associated diarrhoea is predominately a disease of the elderly who have undergone antibiotic treatment. It leads to increased patient morbidity, may contribute to mortality and is often associated with increased hospital stays.

It is well recognized that individual patients can carry this organism without any symptoms of disease. In healthy adults, the rate of asymptomatic colonization is thought to be less than $5 \%$ (Ambrose et al., 1985; Aronsson et al., 1985; Phillips \& Rogers, 1981). However, several studies have shown that rates in hospitalized elderly patients may be much higher (Bender et al., 1986; Brazier et al., 1999).

The effects of antibiotic use on the gut flora are accepted as

Abbreviations: CDAD, Clostridium difficile-associated disease; RVH, Royal Victoria Hospital. the major predisposing factor for the disease; however, there are likely to be many other influential risk factors for the infection.

Many different phenotypic and genotypic methods have been used to fingerprint strains of $C$. difficile in epidemiological studies (Brazier, 2001). The main aims of this investigation were to determine the level of $C$. difficile colonization and disease in a population of elderly patients by routine culture and toxin detection methods, and to use a novel typing method based on the variation of the S-layer proteins from $C$. difficile to compare the strains in the patients and the ward environment. S-typing is a simplified modification of the method based on EDTA-soluble peptides, which was the first-ever method used to show that $C$. difficile was acquired exogenously (Poxton et al., 1984). The present study was part of a major prospective epidemiological study to investigate the use of inferential stochastic modelling to determine control methods for $C$. difficile disease - this is being submitted elsewhere. 


\section{METHODS}

Subjects. During the 17-month period July 1999 to December 2000, 865 geriatric patients were admitted to two wards (designated A and B) of the Royal Victoria Hospital (RVH), Edinburgh. The aim was to collect faecal specimens with informed consent from all patients admitted as soon as possible after admission, and further specimens were collected weekly. Patient information relating to age, antibiotic therapy and symptoms of diarrhoea was collected. C. difficile-associated diarrhoea was judged clinically and defined as at least one episode of diarrhoea (more than three loose stools per day) within the period of 2 weeks prior to and/or 2 weeks after a culture-positive stool specimen was identified. The ages of the patients from whom specimens were collected ranged from 62 to 101 years.

Faecal specimens. Faecal specimens were collected and investigated for the presence of $C$. difficile, irrespective of whether the patient had symptoms of diarrhoea. Fresh faecal specimens were transported to the laboratory as quickly as possible (during a working day) and investigated for the presence of $C$. difficile by culture and detection of toxins A and/or B directly in faecal samples.

Culture of $\boldsymbol{C}$. difficile directly from faecal specimens. A standard loopful (approx. $0.2 \mathrm{~g}$ ) of fresh faecal material was plated directly to prereduced cycloserine-cefoxitin egg yolk (CCEY) agar (Brazier, 1993). The cultures were incubated under anaerobic conditions $(10 \%$ hydrogen, $10 \% \mathrm{CO}_{2}, 80 \%$ nitrogen) at $37^{\circ} \mathrm{C}$ for $24-48 \mathrm{~h}$ and $\mathrm{C}$. difficile was identified by Gram stain, characteristic smell, colonial morphology and chartreuse fluorescence under UV light $(\lambda=365 \mathrm{~nm})$. Following subculture to blood agar, identity was confirmed by the above together with characteristic motility. Any atypical cultures were examined by gas chromatography for the characteristic volatile fatty acid products, including caproic and isocaproic acids (Poxton et al., 1996).

Detection of C. difficile directly in faecal samples by ELISA. Batches of faecal samples were tested retrospectively after storage at $-20^{\circ} \mathrm{C}$ for up to 60 days using the Techlab C. difficile TOX A/B ELISA tests kit. The kit was used in accordance with the manufacturer's instructions.

Toxin-producing potential of $C$. difficile isolates from patients in wards $\mathbf{A}$ and $\mathbf{B}$, the RVH. Isolates of $C$. difficile were grown in brain heart infusion/proteose peptone medium (Brettle et al., 1982) at $37^{\circ} \mathrm{C}$ under anaerobic conditions for 5 days for the detection of toxin. The Techlab C. difficile toxin A/B ELISA test kit was used to detect toxin(s) from pure $C$. difficile cultures.

Isolation and identification of $\boldsymbol{C}$. difficile from the environment of wards $\mathbf{A}$ and $\mathbf{B}$, the RVH. Inanimate surfaces and objects in wards $\mathrm{A}$ and $\mathrm{B}$ were sampled for contamination with $C$. difficile. Many surfaces were sampled; these included floors, commodes, toilet seats and areas within the sluice-rooms. Where practicable, samples were collected using $55 \mathrm{~mm}$ contact plates (Bibby Sterilin) with pre-reduced CCEY agar (Brazier, 1993). After sampling, the plates were stored in an anaerobic atmosphere for transport to the laboratory and incubated at $37^{\circ} \mathrm{C}$ for up to 5 days. Sterile plain cotton wool swabs (Greiner Labortechnik) were also used to sample inanimate surfaces, such as door handles, toilet handles and taps. The swabs were moistened in sterile distilled water before sampling. The swabs were transported to the laboratory where they were inoculated to $4 \mathrm{ml}$ fastidious anaerobe broth supplemented with cholic acid sodium salt and Modified $C$. difficile Selective Supplement (Oxoid). The broth cultures were incubated at $37^{\circ} \mathrm{C}$ for 5 days under anaerobic conditions.

A total of 672 samples were taken from ward A: 331 areas were sampled with contact plates and 341 with swabs. From ward B, a total of 676 samples were taken: 344 areas were sampled with contact plates and 332 with swabs.

Typing of $\boldsymbol{C}$. difficile isolates - 'S-typing'. C. difficile isolates from patients in the RVH were typed using a novel phenotypic typing method. The method utilizes the high degree in variation of the molecular masses of the two C. difficile S-layer proteins. The S-layer proteins were extracted using $5 \mathrm{M}$ guanidine hydrochloride and visualized on SDS-PAGE with Coomassie staining. Following visualization on SDS-PAGE, the molecular masses of the S-layer proteins were calculated using Phoretix gel analysis 1-D software. Mark 12 molecular mass standards (Invitrogen) were used as calibrations for the calculation of molecular masses. Each isolate was given a four-digit number based on the molecular mass in $\mathrm{kDa}$ of the two S-layer proteins as described by McCoubrey \& Poxton (2001). This typing method is known as 'Styping'.

Statistical analysis. All statistical analyses (chi-squared test, MannWhitney U test and logistic regression modelling) were carried out with SPSS software.

\section{RESULTS}

\section{Detection of $C$. difficile by culture and detection of C. difficile toxins A and B}

A total of 1003 specimens were obtained with informed consent from 390 ( $45 \%)$ of the 865 patients admitted to the geriatric unit. C. difficile was detected by culture from 206 (20\%) of the 1003 specimens. These 206 specimens came from $100(26 \%)$ patients. Toxins A and/or B were detected in $52(13 \%)$ of the 390 patients. Of these 52 patients, 18 tested positive for toxin only and the remaining 34 patients were also positive by culture for $C$. difficile. These findings for the two different wards are summarized in Table 1 , and show that C. difficile was detected in a total of 118 (30\%) of the 390 patients investigated. Of these 390 patients, $17 \%$ were positive by culture only, $9 \%$ were positive by both culture and toxin tests, and toxin only was detected in $5 \%$ of patients.

When the results for wards $\mathrm{A}$ and $\mathrm{B}$ were compared, the overall level of detection of $C$. difficile by culture and/or toxin from patients in wards A and B was 58 (27\%) and $60(34 \%)$, respectively. The level of detection of $C$. difficile was not significantly different between the two wards $\left(\chi^{2}\right.$ test, $P=0 \cdot 14)$.

Only $41 \%$ of patients who tested negative for $C$. difficile had been exposed to antibiotics, compared to $67 \%$ of patients from whom $C$. difficile was detected (Odds ratio 2.52; $95 \%$ confidence interval $1 \cdot 60-4 \cdot 16)$. Patients who tested positive for $C$. difficile toxin(s) were significantly older than those who were negative for toxin(s) (mean age 85.3 versus 82.2 , $P=0 \cdot 001)$.

\section{Incidence of diarrhoea in patients who tested positive for $\boldsymbol{C}$. difficile}

Of the patients from whom $C$. difficile was detected, $49 \%$ had diarrhoea, compared to $39 \%$ without any evidence of $C$. 
Table 1. Culture/toxin status of patients investigated for $C$. difficile by culture and toxin detection methods

\begin{tabular}{|llcc|}
\hline $\begin{array}{l}\text { Positive result in } \\
\text { detection test }\end{array}$ & \multicolumn{3}{c|}{ No. (\%) of patients with a positive result } \\
\cline { 2 - 4 } & $\begin{array}{l}\text { Ward A } \\
\boldsymbol{n}=\mathbf{2 1 3}\end{array}$ & $\begin{array}{c}\text { Ward B } \\
\boldsymbol{n}=\mathbf{1 7 7}\end{array}$ & $\begin{array}{c}\text { Total } \\
\boldsymbol{n}=\mathbf{3 9 0}\end{array}$ \\
\hline Culture only & $36(17)$ & $30(17)$ & $66(17)$ \\
Culture and toxin & $12(6)$ & $22(12)$ & $34^{*}(9)$ \\
$\begin{array}{l}\text { Toxin only } \\
\text { Total }\end{array}$ & $10(4 \cdot 5)$ & $8(4 \cdot 5)$ & $18(5)$ \\
& $\mathbf{5 8 ( 2 7 )}$ & $\mathbf{6 0 ( 3 4 )}$ & $\mathbf{1 1 8 ( 3 0 )}$ \\
\hline
\end{tabular}

${ }^{*}$ Culture and toxin may not have been detected in the same specimen.

difficile. Seventy-nine per cent of patients who were cultureand toxin-positive had diarrhoea compared to less than a third of the patients who tested positive by culture only. We have defined Clostridium difficile-associated disease (CDAD) as symptomatic patients from whom both $C$. difficile and its associated toxin(s) were detected. From this definition, $4 \%$ $(8 / 213)$ of patients from ward A and $11 \%(19 / 177)$ from ward $\mathrm{B}$ were determined as suffering from CDAD. However, up to a further $7 \%$ (15 patients) from ward A and $9 \%$ (15 patients) from ward B with diarrhoea may have been suffering from $C$. difficile-related symptoms as they harboured the organism but toxin was not detected. No other enteric pathogen was isolated from any patient during the study.

\section{S-typing of $C$. difficile isolates from patients in wards $A$ and $B$, the RVH}

A total of 206 isolates from 100 patients in the RVH were subjected to S-typing and each isolate was given an S-type number. A total of seven distinct S-types were identified within the collection of isolates from patients in wards A and B. Three isolates from a single patient produced an atypical pattern with only one major S-layer protein -which was deemed untypable (UT). In addition to the SDS-PAGE analysis of the S-layer protein profiles, the $C$. difficile isolates were tested for their ability to produce $C$. difficile toxin(s) in culture by the use of the Techlab ELISA test kit for toxins A and/or B. This revealed that within S-type 5236, some isolates produced toxin(s) and other isolates did not. This allowed further differentiation within S-type 5236, therefore a total of eight different S/toxin-types were identified, with a further toxigenic UT strain. The S-layer protein profiles from representative isolates of each of the seven S-types identified and the UT strain are shown in Fig. 1. Details of the S-type, the toxigenic potential and the frequency of isolation are summarized in Table 2.

Toxigenic S-type 5236 was isolated from $78 \%$ patients and accounted for 150 of the 206 isolates from patients in the $\mathrm{RVH}$. It was evident from the data that toxin-producing Stype 5236 was the endemic strain of $C$. difficile colonizing the

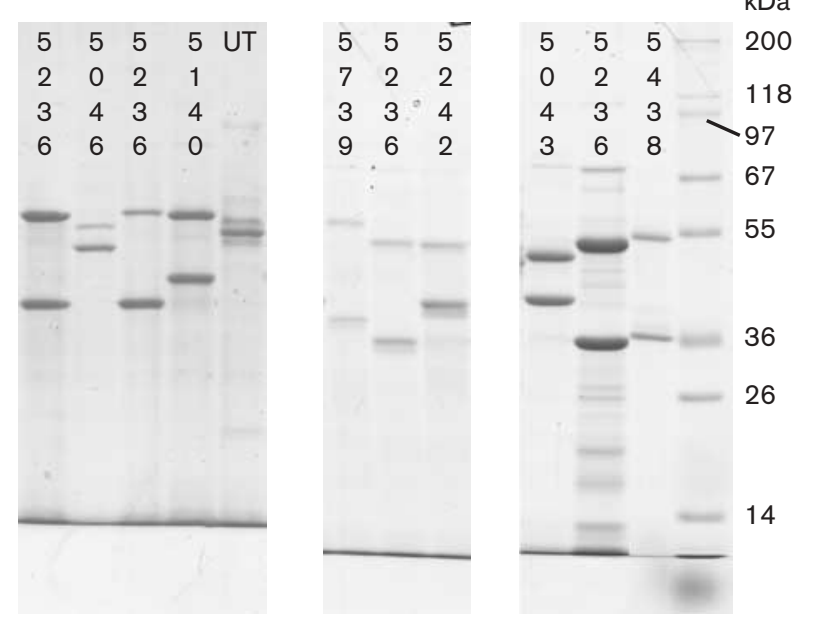

Fig. 1. SDS-PAGE to illustrate the S-layer protein profiles of 11 clinical isolates of $C$. difficile representing each of the nine types identified. These include the seven distinct S-types (S-type designation is at the top of each track), an untypable (UT) strain and examples of toxigenic and non-toxigenic '5236' type. A '5236' is included on each panel, with the one on the right-hand panel being an example of a non-toxigenic variant.

Table 2. Frequency of isolation and the toxigenic potential of each of the S-types isolated

\begin{tabular}{|llcc|}
\hline S-type & Toxigenicity & $\begin{array}{c}\text { No. }(\%) \text { of } \\
\text { isolates }\end{array}$ & $\begin{array}{c}\text { No./\%* of } \\
\text { patients }\end{array}$ \\
\hline 5236 & Toxigenic & $150(73)$ & 78 \\
5236 & Non-toxigenic & $8(4)$ & 4 \\
5242 & Toxigenic & $27(13)$ & 16 \\
5739 & Toxigenic & $1(0 \cdot 5)$ & 1 \\
5438 & Toxigenic & $5(2)$ & 5 \\
5140 & Toxigenic & $5(2)$ & 3 \\
5046 & Non-toxigenic & $6(3)$ & 6 \\
5043 & Toxigenic & $1(0 \cdot 5)$ & 1 \\
UT $\dagger$ & Toxigenic & $3(1 \cdot 5)$ & 1 \\
Total & & $\mathbf{2 0 6}$ & \\
\hline
\end{tabular}

${ }^{*}$ One hundred patients in study. Total percentage is more than 100 as 14 patients were colonized with more than one S-type of $C$. difficile.

$\dagger$ This toxigenic strain did not produce the two distinct S-proteins on SDS-PAGE, thus an S-type number was not designated.

patients in wards A and B, the RVH. The other S-types were isolated infrequently and were associated with very small numbers of patients.

\section{Incidence of $C$. difficile in the environment of patients in wards $A$ and $B$, the RVH}

The wards were mirror images of each other and consisted of four main bays with six beds in each, and each ward had five side-rooms. Each bay had its own designated toilet area and 
the bathroom and shower-room facilities were shared between bays. Each of the side-rooms had its own designated toilet and bathroom facilities. In addition, each ward had a sluice-room and kitchen. C. difficile was isolated from $23 \%$ of samples taken by contact plates and $4 \%$ of samples using swabs.

The number of samples taken and the number of areas that tested positive from each room in wards $A$ and $B$ are summarized in Table 3. In ward A, C. difficile was most frequently isolated from the sluice-room, followed by the toilet areas associated with side-rooms. In ward B, the most frequent isolations were from toilet areas associated with side-rooms and the side-rooms themselves. The main toilet areas and sluice-room in ward B also had relatively high isolation levels, $17 \%$ and $15 \%$ of areas, respectively. The data in Table 3 show an overall level of isolation of $C$. difficile from the environment of ward A at $7 \%$ and from ward B at $20 \%$. Correspondingly, all rooms of ward B showed higher levels of isolation of $C$. difficile than the equivalent rooms in ward A. Consistent with the overall results for wards $A$ and $B$, the level of isolation of $C$. difficile in the side-rooms of ward B was much higher than those isolation levels from the side-rooms of ward A.

Of the areas sampled, $C$. difficile was most frequently isolated from floors; $29 \%$ of all floors from which samples were taken throughout wards A and B yielded a positive culture of $C$. difficile. Of the floors sampled, the toilet floors and the sluiceroom floor yielded the highest isolation levels, with $40 \%$ of samples taken from toilet floors and $60 \%$ of samples from sluice-room floors yielding a positive result. Other areas with relatively high isolation levels included toilet seats $(17 \%)$, commodes $(11 \%)$ and toilet handles $(8 \%)$, and three of the five toilet cisterns tested from ward B gave culture-positive results for $C$. difficile. Numerous other objects were sampled and gave positive results; amongst these, the paper-towel dispensers from the ward B kitchen, side-room 8 in ward B and bay 3 in ward B all tested positive for $C$. difficile. Two of six windowsills in ward B produced positive results, as did $25 \%$ of the shelves and cupboard tops from the sluice-rooms and bathrooms of ward $\mathrm{B}$.

A total of 203 environmental isolates from 168 areas sampled were S-typed. All isolates tested were identified as belonging to one of the three major S-types 5236 (91\%), 5242 (7\%) and $5438(2 \%)$. Most of these isolates were tested for toxin production and all were positive. Isolation rates of S-types 5236 and 5242 from the environment were plotted against isolation rates of these types from patients for each ward. The relationships were very complex (data not shown). In one ward (A) where there were peaks and troughs of isolations from both patients and environment, peak isolation rates from the environment seemed to precede peak levels in the patient. However, in the other ward, the relationships were not so obvious. Here the levels in the patients were much more constant but with peaks of isolations from the environment, possibly reflecting cleaning levels; however, this parameter was not measured quantitatively.

\section{DISCUSSION}

This study of a geriatric unit investigated patients for colonization and infection with $C$. difficile, and revealed a $30 \%$ incidence of colonization with $C$. difficile in the patients investigated. Reported levels of isolation of $C$. difficile from different groups of hospitalized patients are highly variable. In close agreement with this study, McFarland et al. (1989) reported the isolation of $C$. difficile from $26 \%$ of patients in a general medical ward, and Gerding et al. (1986) reported that in a group of geriatric patients with diarrhoea, $30 \%$ harboured the organism. In an investigation of six different Welsh hospitals, $16 \cdot 5 \%$ elderly patients acquired $C$. difficile during their hospital stay. Two of the Welsh hospitals investigated reported that $C$. difficile was not isolated and the range of acquisition by patients in the other four hospitals was 4-24\% (Brazier et al., 1999). In a chronic care facility for

Table 3. Number of samples taken and the number of samples positive for the culture of $C$. difficile from each room in wards $A$ and $B$

\begin{tabular}{|c|c|c|c|c|}
\hline \multirow[t]{2}{*}{ Area } & \multicolumn{2}{|c|}{ Ward A } & \multicolumn{2}{|c|}{ Ward B } \\
\hline & Total samples & $\begin{array}{c}\text { No. }(\%) \text { of } \\
\text { samples positive } \\
\text { for } C \text {. difficile }\end{array}$ & Total samples & $\begin{array}{c}\text { No. }(\%) \text { of } \\
\text { samples positive } \\
\text { for C. difficile }\end{array}$ \\
\hline Main patient room & 160 & $9(6)$ & 138 & $12(9)$ \\
\hline Main toilet areas & 235 & $16(7)$ & 174 & $30(17)$ \\
\hline Bathrooms & 78 & $3(4)$ & 64 & $6(9)$ \\
\hline Shower-rooms & 41 & $3(7)$ & 40 & $3(7 \cdot 5)$ \\
\hline Sluice-rooms & 60 & $8(13)$ & 71 & $11(15)$ \\
\hline Side-rooms: main area & 53 & $4(7 \cdot 5)$ & 80 & $32(40)$ \\
\hline Side-rooms: toilet area & 43 & $4(9)$ & 98 & $41(42)$ \\
\hline Kitchen & 2 & 0 & 11 & $2(18)$ \\
\hline Total & 672 & $48(7 \cdot 1)$ & 676 & $137(20)$ \\
\hline
\end{tabular}


the elderly, approximately $30 \%$ of patients were found to harbour C. difficile. Of the patients studied, $28 \%$ were toxinpositive and $33 \%$ were culture-positive (Bender et al., 1986). This compares well with the $27 \%$ of culture-positive and $14 \%$ of toxin-positive patients in the RVH. It also correlates with the overall detection level of $30 \%$ from patients in the RVH.

Toxin was not detected in the faecal samples of $54 \%$ of patients who were colonized with $C$. difficile. It is well recognized that toxin is often not present in the faeces of asymptomatic, colonized patients. This may be because the toxin is not produced in these patients in detectable quantities, or that patients are colonized by non-toxigenic strains.

From our study, $4 \%$ of patients from ward A and $11 \%$ from ward $\mathrm{B}$ were determined as suffering from CDAD. There were, however, a significant number of symptomatic patients from whom $C$. difficile was detected by culture or toxin testing, and although they did not fulfil the criteria for CDAD, their symptoms may be related to infection with $C$. difficile.

Sampling of the environment of the wards in the RVH indicated that ward B was much more heavily contaminated than ward A. C. difficile was isolated from $7 \%$ of environmental surfaces sampled in ward A and $20 \%$ of those surfaces sampled in ward B. These findings compare with a study which isolated $C$. difficile from $15 \%$ of environmental surfaces in a chronic care ward for the elderly (Bender $e t$ al., 1986) and an investigation by Al Saif \& Brazier (1996) which isolated C. difficile from $20 \%$ of inanimate objects from various hospital environments.

The areas with the highest isolation levels of $C$. difficile were toilet areas, sluice-rooms and side-rooms. As toilet areas and sluice-rooms are most likely to become contaminated by faecal material infected with $C$. difficile, this finding is not surprising. Patients who were symptomatic carriers of the organism often occupied the side-rooms and therefore contamination of such an environment is inevitable. McFarland et al. (1989) isolated C. difficile from $36 \%$ of floors, $18 \%$ of toilets and $38 \%$ of commodes, and Samore et al. (1996) also isolated the organism from $48 \%$ of floors, $41 \%$ of commodes and $33 \%$ of toilets. C. difficile was most frequently isolated from the floor of the $\mathrm{RVH}$, and toilets and commodes were also frequently contaminated. The high levels of contamination of floors may be due to the initial contamination and subsequent movement of spores from place to place on the feet of staff, patients and visitors. Interestingly, McFarland et al. (1989) and Samore et al. (1996) isolated C. difficile from windowsills at levels of $30 \%$ and $38 \%$, respectively. C. difficile was isolated from $20 \%$ of windowsills sampled in the RVH. The organism was also isolated from shelves and cupboard tops. Areas such as these collect dust and may not be subject to cleaning as frequently as other areas, hence the spores collect and provide a reservoir of the organism. Fekety et al. (1981) reported the recovery of $C$. difficile from an intentionally contaminated surface for up to 5 months, demonstrating the ability of the organism to survive.

Environmental sampling of the study area utilized both contact plates and swabs. Samples taken by contact plates yielded a much higher isolation level of $C$. difficile than the samples taken with swabs. In a study comparing the relative merits of contact plates and swab methods, it was stated that contact plates were the preferred method of sampling and that they were better than swabs (Buggy et al., 1983). It is therefore probably the case that the lower yields from the swabs were a result of the sampling method rather than the areas sampled.

S-typing is extremely simple to perform and comparison of isolates is exceptionally easy by the simple 2-band pattern produced. It was developed from one of the earliest and wellproven phenotyping methods, EDTA-extracted proteins, which has been performed extensively throughout the world. S-typing has been compared with PCR ribotyping and there was excellent correlation between the two methods (McCoubrey, 2002). A combination of S-typing and toxin testing of isolates from patients and the environment of wards A and B of the RVH identified a total of eight distinct S/ toxin-types (with one untypable) from the 206 patient and 203 environmental isolates tested during the study. The endemic strain, toxigenic S-type 5236, colonized threequarters of all the patients from whom $C$. difficile was isolated. This endemic S-type corresponded to PCR ribotype 1 (McCoubrey, 2002), which is the endemic strain in the rest of the UK (Brazier et al., 1997). The seven other S/toxin-types and the untypable colonized a comparatively small number of patients. These findings were similar to those from a study carried out by Fawley \& Wilcox (2001). Their study investigated the patients and the environment of two elderly general medicine wards over a 22 -month period.

The toxigenic S-type 5236 that was endemic in patients in the $\mathrm{RVH}$ also accounted for the majority of the RVH environmental isolates. Two of the other eight S/toxin-types (5242/T and $5438 / \mathrm{T}$ ) that were isolated from patients were also isolated from the environment, suggesting a possible association between the S-types isolated from patients and their environment.

Other studies have made associations between the contamination of the environment with specific strains and colonization of patients with the same strains. Cohen et al. (2000) showed that the AP-PCR types identified from patients in a geriatric and general medicine unit correlated closely with the types identified from the environment of these patients.

Interestingly, S-type 5438 was isolated from two patients in ward A and then several months later from three patients in ward B. All the patients colonized with this strain were housed in different bays, suggesting that contamination of the environment may not have been directly responsible if cross-infection occurred with this strain. In the study by Fawley \& Wilcox (2001), they reported that a particular 
genotype was not isolated from the environment until the sixth patient in a cluster of cases became symptomatic. These data from both the RVH and Fawley \& Wilcox (2001) suggest that an increase in the number of colonized patients can lead to an increase in the levels of environmental contamination with the colonizing strain. Fawley \& Wilcox (2001) also suggested that initial cross-infection from patient to patient or from staff to patient may occur before heavy environmental contamination occurs and causes further crosscontamination. This may perhaps explain the transfer of Stype 5438 between patients in different bays within ward A. However, other sources such as commodes and equipment that are shared between bays within wards may be the common source of infection.

Due to the endemic nature of S-type 5236 in both the environment and the patients, it was not possible to determine whether environmental contamination or patient-patient and staff-patient spread was the main source of cross-infection in wards A and B, the RVH. Fawley \& Wilcox (2001) encountered similar difficulties in determining the source of infection. They identified six different genotypes from the environment; however, only two of these were also isolated from patients and the endemic genotype corresponding to PCR ribotype 1 accounted for $92 \%$ of the environmental $C$. difficile isolates.

With the aid of a novel typing technique this study illustrates the endemic nature of $C$. difficile in a geriatric population and the degree to which their environment is contaminated. Much epidemiological evidence for the role of environmental contamination in the transfer of infection has been demonstrated; however, the relative importance of environmental contamination, patient-patient and staff-patient cross-contamination has to be determined. This stresses the paramount importance of infection control, including good hand-washing procedures by all health workers. It emphasizes the need for good ward cleansing practices at all times in order to reduce the opportunity for cross-contamination and the requirement for an effective disinfectant against $C$. difficile spores.

\section{ACKNOWLEDGEMENTS}

The authors would like to thank the Scottish Executive Chief Scientist Office (Grant No. K/OPR/2/2/D343) for supporting this research. We are grateful to $\mathrm{Mr}$ Robert Brown for his technical advice and to the nursing staff of the Royal Victoria Hospital for their cooperation during the study.

\section{REFERENCES}

Al Saif, N. \& Brazier, J. S. (1996). The distribution of Clostridium difficile in the environment of South Wales. J Med Microbiol 45, 133-137.

Ambrose, N. S., Johnson, M., Burdon, D. W. \& Keighly, M. R. B. (1985). The influence of single dose intravenous antibiotics on faecal flora and emergence of Clostridium difficile. J Antimicrob Chemother 15, 319-326.
Aronsson, B., Mollby, R. \& Nord, C. E. (1985). Antimicrobial agents and Clostridium difficile in acute enteric disease: epidemiological data from Sweden, 1980-1982. J Infect Dis 151, 476-481.

Bender, B. S., Bennett, R., Laughon, B. E., Greenough, W. B., Gaydos, C., Sears, S. D., Forman, M. S. \& Bartlett, J. G. (1986). Is Clostridium difficile endemic in chronic-care facilities? Lancet ii, 11-13.

Brazier, J. S. (1993). Role of the laboratory in investigations of Clostridium difficile diarrhoea. Clin Infect Dis 16 (Suppl. 4), S228-S233.

Brazier, J.S. (2001). Typing of Clostridium difficile. Clin Microbiol Infect 7, 428-431.

Brazier, J. S., Mulligan, M. E., Delmée, M., Tabaqchali, S. \& the International Clostridium difficile study group (1997). Preliminary findings of the international typing study on Clostridium difficile. Clin Infect Dis 25 (Suppl. 2), S199-201.

Brazier, J. S., Fitzgerald, T. C., Hosein, I., Cefai, C., Looker, N., Walker, M., Buschell, A. C. \& Rooney, P. (1999). Screening for carriage and nosocomial acquisition of Clostridium difficile by culture: a study of 284 admissions of elderly patients to six general hospitals in Wales. J Hosp Infect 43, 317-319.

Brettle, R. P., Poxton, I. R., Murdoch, J. M., Brown, R., Byrne, M. D. \& Collee, J. G. (1982). Clostridium difficile in association with sporadic diarrhoea. Br Med J 284, 230-233.

Buggy, B. P., Wilson, K. H. \& Fekety, R. (1983). Comparison of methods for recovery of Clostridium difficile from an environmental surface. J Clin Microbiol 18, 348-352.

Cohen, S. H., Tang, Y. J., Rahmani, D. \& Silva, J. (2000). Persistence of an endemic (toxigenic) isolate of Clostridium difficile in the environment of a general medicine ward. Clin Infect Dis 30, 952-953.

Fawley, W. N. \& Wilcox, M. H. (2001). Molecular epidemiology of endemic Clostridium difficile infection. Epidemiol Infect 126, 343-350.

Fekety, R., Kim, K. H., Brown, D., Batts, D. H., Cudmore, M. \& Silva, J. (1981). Epidemiology of antibiotic-associated colitis; isolation of Clostridium difficile from the hospital environment. Am J Med 70, 906-908.

Gerding, D. N., Olson, M. M., Peterson, L. R., Teasley, D. G., Gebhard, R. L., Schwartz, M. L. \& Lee, J. T. (1986). Clostridium difficile-associated diarrhoea and colitis in adults. Arch Intern Med 146, 95-100.

McCoubrey J. (2002). The epidemiology of Clostridium difficile in a geriatric unit. $\mathrm{PhD}$ thesis, University of Edinburgh.

McCoubrey, J. \& Poxton, I. R. (2001). Variation in the surface layer proteins of Clostridium difficile. FEMS Immunol Med Microbiol 31, 131-135.

McFarland, L. V., Mulligan, M. E., Kwok, R. \& Stamm, W. E. (1989). Nosocomial acquisition of Clostridium difficile infection. N Engl J Med 320, 204-210.

Phillips, K. D. \& Rogers, P. A. (1981). Rapid detection and presumptive identification of Clostridium difficile by p-cresol production on a selective medium. J Clin Pathol 34, 642-644.

Poxton, I. R., Aronsson, B., Mollby, R., Nord, C.-E. \& Collee, J. G. (1984). Immunochemical fingerprinting of Clostridium difficile strains isolated from an outbreak of antibiotic associated colitis and diarrhoea. J Med Microbiol 17, 317-324.

Poxton, I. R., Brown, R., Fraser, A. G. \& Collee, A. G. (1996). Enteropathogenic clostridia and Clostridium botulinum. In Mackie and McCartney's Practical Medical Microbiology, 14th edn, pp. 537547. Edited by J. G. Collee, A. G. Fraser, B. P. Marmion \& A. Simmons. Edinburgh: Churchill Livingstone.

Samore, M. H., Venkataraman, L., DeGirlami, P. C., Arbeit, R. D. \& Karchmar, A. W. (1996). Clinical and molecular epidemiology of sporadic and clustered cases of nosocomial Clostridium difficile diarrhea. Am J Med 100, 32-40. 